\title{
Effective-liquid approach to the generalized Onsager theories of the isotropic-nematic transition of hard convex bodies
}

\author{
José A. Cuesta and Carlos F. Tejero \\ Facultad de Ciencias Físicas, Universidad Complutense de Madrid, 28040 Madrid, Spain \\ Hong $\mathrm{Xu}$ \\ Van't Hoff Laboratorium, Rijkuniversiteit te Utrecht, NL-3508TB Utrecht, The Netherlands
}

\author{
Marc Baus \\ Physique Statistique et Plasmas, Code Postal 231, Université Libre de Bruxelles, B-1050 Brussels, Belgium
}

(Received 3 May 1991)

\begin{abstract}
Recent attempts to generalize the classical Onsager theory of nematic ordering to finite-density systems of finite-length hard convex bodies are related and compared. It is pointed out that, although good results can be obtained in three-dimensions (3D), in two dimensions (2D) the underlying factorization approximation of the radial and angular variables always implies a second-order isotropic-nematic transition instead of the crossover from a weakly first-order transition to a continuous (Kosterlitz-Thouless) transition as seen in the simulations. The quantitative agreement with the simulations is also much poorer in $2 \mathrm{D}$ than in $3 \mathrm{D}$. On the contrary, for large spatial dimensions these theories become exact.
\end{abstract}

PACS number(s): 61.30.By, 64.70.Md, 05.70.Fh

It has been shown recently that the positional freezing of hard-sphere (HS) systems can be adequately described within the effective-liquid approximation (ELA) to the density-functional theory (DFT) of nonuniform systems [1]. Although it has also been shown that the extension to softer potentials is not straightforward [2], it can be hoped that for hard-core interactions a similar approach can still be used for the orientational freezing seen in recent simulation studies $[3,4]$. A number of such studies have in fact already been performed [5-12]. It is the purpose of this Brief Report to point out (i) the relation between some of these recent attempts and the DFT of freezing within the ELA and (ii) a fundamental difficulty in the two-dimensional (2D) version of these theories.

Let $\phi=F / V \rho$ denote the intrinsic Helmholtz free energy $(F)$ per particle of a system whose local density averaged over the volume $V$ is $\rho$. This free energy $\phi$ can always be split into an ideal part $\left(\phi_{\text {id }}\right)$ and an excess contribution $\left(\phi_{\mathrm{ex}}\right)$ as $\phi=\phi_{\mathrm{id}}+\phi_{\mathrm{ex}}$ where

$$
\begin{aligned}
& \beta \phi_{\mathrm{id}}[\rho]=\frac{1}{V \rho} \int d \mathbf{x} \rho(\mathbf{x}) {[\ln \Lambda \rho(\mathbf{x})-1], } \\
& \beta \phi_{\mathrm{ex}}[\rho]=-\frac{1}{V \rho} \int d \mathbf{x} \int d \mathbf{x}^{\prime} \rho(\mathbf{x}) \rho\left(\mathbf{x}^{\prime}\right) \\
& \times \int_{0}^{1} d \lambda(1-\lambda) c\left(\mathbf{x}, \mathbf{x}^{\prime} ;[\lambda \rho]\right),
\end{aligned}
$$

with $\beta=1 / k_{B} T$ the inverse temperature, while $\mathbf{x}=(\mathbf{r}, \mathbf{u})$ denotes both the translational $(\mathbf{r})$ and orientational $(\mathbf{u})$ degrees of freedom. The normalization is such that $\int d \mathbf{r}=V$ and $\int d \mathbf{u}=1$. The square brackets in (1) and (2) indicate a functional dependence on the one-body density $\rho(\mathbf{x})$ with $\int d \mathbf{x} \rho(\mathbf{x})=V \rho$. In what follows we will only consider molecules with a cylindrical symmetry axis and an up-down symmetry in which case $u$ can always be tak- en to be a unit vector along this axis. Both $2 \mathrm{D}$ and $3 \mathrm{D}$ systems can be considered by interpreting $\mathbf{r}$ and $\mathbf{u}$, respectively, as $D$-dimensional and $(D-1)$-dimensional vectors $(D=2,3)$ and $\Lambda$ in (1) as the thermal wavelength to the power $D$. The total free energy can then be obtained by adding to $\phi$ the contribution of the external field which confines the system to the volume $V$ and eventually destroys some of the symmetries of the underlying Hamiltonian. In the thermodynamic limit the externalfield contribution can be omitted and the symmetrybreaking features can be imposed directly on $\rho(\mathbf{x})$. For the isotropic $(I)$-nematic $(N)$ transition under consideration here, only orientational order is present and we can put $\rho(\mathbf{x})=\rho h(\mathbf{u})$ with $h(\mathbf{u})$ the normalized $\left[\int d \mathbf{u} h(\mathbf{u})=1\right]$ orientational distribution. Equations (1) and (2) read then

$$
\begin{aligned}
& \beta \phi_{\mathrm{id}}[h]=\ln (\Lambda \rho)-1+ \int d \mathbf{u} h(\mathbf{u}) \ln h(\mathbf{u}), \\
& \beta \phi_{\mathrm{ex}}[h]=-\rho \int d \mathbf{u} \int d \mathbf{u}^{\prime} h(\mathbf{u}) h\left(\mathbf{u}^{\prime}\right) \\
& \times \int_{0}^{1} d \lambda(1-\lambda) \\
& \times \int d \mathbf{r} c\left(\mathbf{r} ; \mathbf{u}, \mathbf{u}^{\prime} ;[\lambda h]\right),
\end{aligned}
$$

where we took already into account that for the translationally invariant $I$ and $N$ phases the direct correlation function (DCF) of (2) can be written as $c\left(\mathbf{x}, \mathbf{x}^{\prime}\right)=c\left(\mathbf{r}-\mathbf{r}^{\prime} ; \mathbf{u}, \mathbf{u}^{\prime}\right)$. The DFT of these phases can then be completed by parametrizing $h(\mathbf{u})$ in terms of known functions [11] and minimizing $\phi$ with respect to these (order) parameters for a given DCF. The latter function is however not known and different strategies can be followed in order to approximate its contribution to (4). One can perform a virial expansion as in the original Onsager theory [13] and stop at third order as recent- 
ly proposed by Tjipto-Margo and Evans [12]. Or, one can expand the DCF around that of an isotropic reference fluid as proposed by Singh and Singh [5], and Marko [7] in which case the isotropic reference fluid DCF can be computed numerically from a known integral equation as proposed by Perera, Patey, and Weis [9]. Finally, one can also transpose the DFT of positional freezing within the ELA to this case as proposed by Baus, Colot, Wu, and $\mathrm{Xu}$ [6] for 3D and by Cuesta, Tejero, and Baus [11] for 2D systems. The ELA amounts [1] to replace in (4) the exact DCF by the DCF of an isotropic fluid evaluated at an effective (uniform and isotropic) density $\bar{\rho}$ :

$$
c\left(\mathbf{r} ; \mathbf{u}, \mathbf{u}^{\prime} ;[h]\right)=c_{I}\left(\mathbf{r} ; \mathbf{u}, \mathbf{u}^{\prime} ; \bar{\rho}[h]\right),
$$

where, as indicated in (5), $c_{I}(\bar{\rho})$ is a function of $\bar{\rho}$, which itself remains in general a functional of $\rho h(\mathbf{u})$, i.e., $\bar{\rho}=\bar{\rho}[h]$. Since even for hard convex bodies (HCB) (such as ellipsoids or spherocylinders $[3,4]$ ) the isotropic fluid DCF is still unknown the latter can (following the original suggestion of Pynn [14]) be further approximated in terms of the DCF of a fluid of HS of the same volume as the volume $v_{0}$ of the $\mathrm{HCB}$ and with a $\mathrm{HS}$ diameter equal to the contact distance, $\sigma\left(\widehat{\mathbf{r}} ; \mathbf{u}, \mathbf{u}^{\prime}\right)$, of two $\mathrm{HCB}$ of given orientations $\mathbf{u}$ and $\mathbf{u}^{\prime}$ :

$$
c_{I}^{\mathrm{HCB}}\left(\mathbf{r} ; \mathbf{u}, \mathbf{u}^{\prime} ; \bar{\rho}\right)=c_{\mathrm{HS}}\left(\frac{|\mathbf{r}|}{\sigma\left(\widehat{\mathbf{r}} ; \mathbf{u}, \mathbf{u}^{\prime}\right)} ; \bar{\rho} v_{0}\right),
$$

where $\hat{\mathbf{r}}=\mathbf{r} /|\mathbf{r}|$, and $\bar{\rho} v_{0}$ is the packing fraction. Using (5) and (6) in (4) we obtain an expression for the excess free energy which factorizes the radial and angular variables as

$$
\begin{aligned}
\beta \phi_{\mathrm{ex}}[h]= & \left.-24 \rho v_{0} \int_{0}^{1} d \lambda(1-\lambda) \int_{0}^{\infty} d x x^{2} c_{\mathrm{HS}}\left(x ; v_{0} \bar{\rho}[\lambda h]\right)\right) \\
& \times\left[\int d \mathbf{u} \int d \mathbf{u}^{\prime} h(\mathbf{u}) h\left(\mathbf{u}^{\prime}\right) V_{\mathrm{excl}}\left(\mathbf{u} \cdot \mathbf{u}^{\prime}\right)\right]
\end{aligned}
$$

where

$$
V_{\mathrm{excl}}\left(\mathbf{u} \cdot \mathbf{u}^{\prime}\right)=\frac{1}{24 v_{0}} \int d \hat{\mathbf{r}}\left[\sigma\left(\widehat{\mathbf{r}} ; \mathbf{u}, \mathbf{u}^{\prime}\right)\right]^{3}
$$

is the reduced excluded volume of two HCB of fixed orientations $\mathbf{u}$ and $\mathbf{u}^{\prime}$. Notice that (7) is at once of the generalized Onsager form from which the classical Onsager theory [13] can be recovered by putting the radial bracket of (7) equal to $v_{0} \rho / 2$, i.e., by replacing the HS DCF by its low-density limit $\left[c_{\mathrm{HS}}(x ; 0)=-\Theta(1-x)\right.$ with $\Theta(x)$ the Heaviside step function]. The final implementation of (7) requires still a prescription for the effective density $\bar{\rho}=\bar{\rho}[h]$. In the earlier attempts $[6,11]$ this prescription was taken from geometric considerations extraneous to the modern DFT of freezing [1]. In the latter $\bar{\rho}[h]$ is determined instead from a self-consistency condition between $\phi_{\mathrm{ex}}[h]$ and the excess free energy of the isotropic phase, say $\phi_{\mathrm{ex}}^{0}(\bar{\rho})$. Because of the factorization approximation embodied in (7) there are now two ways to express this condition according to whether one imposes the self-consistency on the total excess free energy or, since the angular part is density independent, on its radial

TABLE I. The isotropic-nematic coexistence of hard ellipsoids $(D=3)$ of aspect ratio $k$ as predicted from Eqs. (9) and (12) and compared to the Monte Carlo (MC) simulation data of Frenkel and Mulder [3]. Here $\eta_{I}=\rho_{I} v_{0}$ denotes the packing fraction of the isotropic phase, $\Delta \eta=\eta_{N}-\eta_{I}, P^{*}=\beta p v_{0}$ the reduced pressure, $\mu_{\mathrm{ex}}^{*}=\beta \mu_{\mathrm{ex}}$ the reduced excess chemical potential, and $q=\left\langle P_{2}(\cos \theta)\right\rangle$ the quadrupole moment of the angular distribution of the nematic at coexistence. All the theoretical results have been obtained using the Carnahan-Starling HS equation of state [6] for $Q_{\mathrm{HS}}(\eta)$, the Maier-Saupe one orderparameter approximation [6] for $h(\mathbf{u})$ and the Berne-Pechukas approximation [6] for the overlap distance $\sigma\left(\widehat{\mathbf{r}} ; \mathbf{u}, \mathbf{u}^{\prime}\right)$ (rescaled in such a way as to restore the exact second virial coefficient [4]). The results shown for Eq. (12) differ slightly from those of Lee [8] because in the latter work $h$ (u) was evaluated numerically yielding a slightly better agreement with the MC results. The results shown for Eq. (9) differ slightly from those of Holyst and Poniewierski [10] because in the latter work a better algorithm for the overlap distance was used together with a modified HS equation of state. All theoretical results have an exact $k$ to $1 / k$ symmetry not shared by the MC results. The best overall agreement is obtained from Eq. (12) which, e.g., for $k=2$ predicts an $I-N$ transition well above the isotropic-solid transition seen in the simulations (the corresponding MC data are shown between brackets).

\begin{tabular}{llccccc}
\hline \hline & $k$ & $\eta_{I}$ & $\Delta_{\eta}$ & $P^{*}$ & $\mu_{\text {ex }}^{*}$ & $q$ \\
\hline \multirow{2}{*}{ MC } & 3 & 0.507 & 0.010 & 9.79 & 25.15 & \\
& $\frac{1}{3}$ & 0.498 & 0.010 & 9.15 & 24.03 & \\
& 2.75 & 0.561 & 0.009 & 15.70 & 35.68 & \\
& $\frac{1}{2.75}$ & 0.545 & 0.014 & 13.45 & 41.69 & \\
& 2 & $(0.620)$ & & $(24.59)$ & $(49.03)$ & \\
Eq. (12) & 3 & $(0.615)$ & & $(23.96)$ & $(48.23)$ & \\
& 2.75 & 0.523 & 0.009 & 10.72 & 28.1 & 0.536 \\
& 2 & 0.557 & 0.008 & 14.00 & 33.7 & 0.522 \\
Eq. (9) & 3 & 0.693 & 0.003 & 50.10 & 88.3 & 0.477 \\
& 2.75 & 0.394 & 0.020 & 3.43 & 12.79 & 0.488 \\
& 2 & 0.417 & 0.019 & 4.06 & 14.08 & 0.480 \\
& 2 & 0.523 & 0.011 & 8.97 & 25.33 & 0.453 \\
\hline \hline
\end{tabular}


part only. In the former case one has $\phi_{\mathrm{ex}}[h]=\phi_{\mathrm{ex}}^{0}(\bar{\rho})$ and (7) reduces to

$$
\beta \phi_{\mathrm{ex}}[h]=Q_{\mathrm{HS}}(\bar{\eta}[h])\left[\int d \mathbf{u} \int d \mathbf{u}^{\prime} V_{\mathrm{excl}}\left(\mathbf{u} \cdot \mathbf{u}^{\prime}\right)\right],
$$

where $Q_{\mathrm{HS}}(\eta)$ is related to the HS compressibility factor, $Z_{\mathrm{HS}}(\eta)$, by

$$
Q_{\mathrm{HS}}(\eta)=\int_{0}^{\eta} d y \frac{Z_{\mathrm{HS}}(y)-1}{y}
$$

and the effective packing fraction, $\bar{\eta}=v_{0} \bar{\rho}$, is determined by the GELA (generalized effective-liquid approximation) equation of Ref. [1], which in the simpler SCELA (selfconsistent effective-liquid approximation) approximation [1] (corresponding to $\bar{\rho}[\lambda h]=\lambda \bar{\rho}[h]$ ) simplifies to

$$
\bar{\rho}[h]=\rho \frac{\int d \mathbf{u} \int d \mathbf{u}^{\prime} h(\mathbf{u}) h\left(\mathbf{u}^{\prime}\right) V_{\mathrm{excl}}\left(\mathbf{u} \cdot \mathbf{u}^{\prime}\right)}{\int d \mathbf{u} \int d \mathbf{u}^{\prime} V_{\mathrm{excl}}\left(\mathbf{u} \cdot \mathbf{u}^{\prime}\right)} .
$$

If, on the contrary, we impose the self-consistency on the radial part only, we obtain $\bar{\rho}[\lambda h]=\lambda \rho$, and (7) reduces to

$$
\beta \phi_{\mathrm{ex}}[h]=Q_{\mathrm{HS}}(\eta)\left[\int d \mathbf{u} \int d \mathbf{u}^{\prime} h(\mathbf{u}) h\left(\mathbf{u}^{\prime}\right) V_{\mathrm{excl}}\left(\mathbf{u} \cdot \mathbf{u}^{\prime}\right)\right]
$$

instead of (9). Notice that (12) is identical to the expression proposed by Lee [8] on the basis of a scaling argument while (9) is identical to the expression proposed by Holyst and Poniewierski [10] within the smoothed density formalism of Tarazona [15]. Both expressions are thus closely related to the DFT within the SCELA approximation. In the low-density limit, $Q_{\mathrm{HS}}(\eta)=\eta / 2$, and both (9) and (12) become identical to the original Onsager expression [13]. For higher densities, $Q_{\mathrm{HS}}(\eta)$ has been implemented differently by different authors $[8,10]$ but we find that for any given HS equation of state the results obtained from (12) are always superior to those of (9). An example of the agreement between (9) and (12) and the computer simulations [3] is given in Table I. It is interesting to observe that both (9) and (12) and the one order-parameter approximation for $h(\mathbf{u})$ proposed elsewhere [11] [and which was used here to test (9) and (12)] all become exact in the limit of very large spatial dimensions $D$ for which we recover [16] the results of Carmesin, Frish and Percus [17]. The resulting $(D=\infty)$ phase diagram is shown in Fig. 1. It can thus be expected that the numerical results obtained from (9) and (12) will improve with increasing $D$ values. One obvious defect of (9) and (12) results from their exact oblate-prolate symmetry, directly induced by the corresponding property of $V_{\mathrm{ex}}\left(\mathbf{u} \cdot \mathbf{u}^{\prime}\right)$, while this symmetry is only approximately born out by the simulations [3]. The major defect of (9) and (12) however is that for $D=2$ they always predict a continuous $I-N$ transition while the simulations of Cuesta and Frenkel [4] show a crossover from a weakly firstorder transition to a continuous (Kosterlitz-Thouless) transition. Indeed it can be shown theoretically, by extending [16] the bifurcation analysis of Kayser and Raveche $[18]$ to (9) and (12), that whenever the radial and angular parts of the excess free energy factorize the resulting transition is second order. This property results

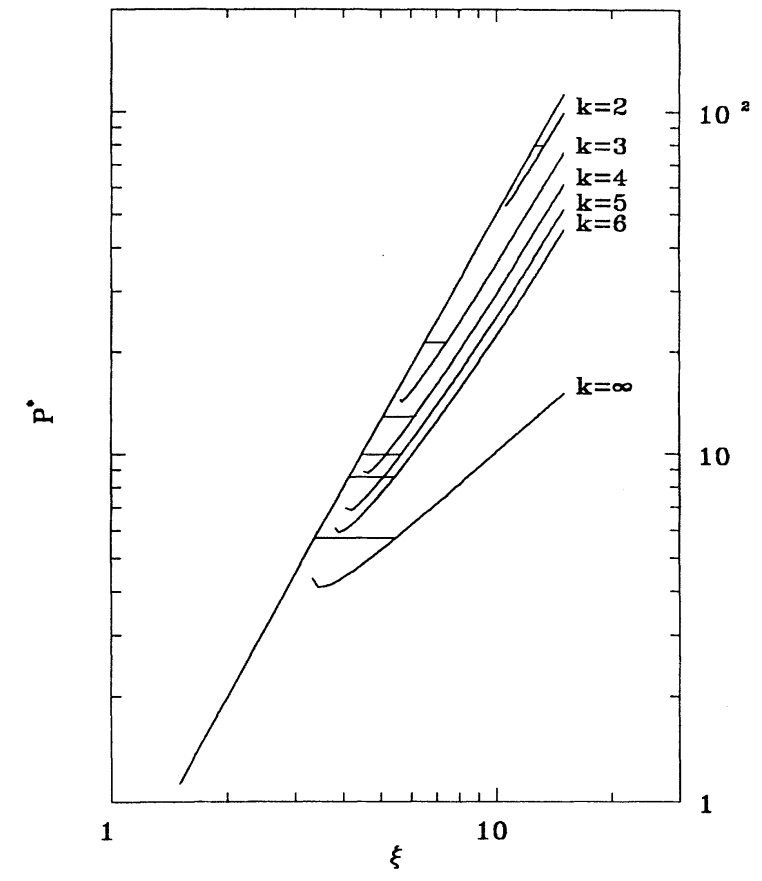

FIG. 1. The phase diagram of hard ellipsoids in the BernePechukas approximation [19] as obtained [16] from (12) in the limit of very large spatial dimensionality $(D=\infty)$. The reduced pressure $P^{*}=\xi \beta P / D \rho$ vs the reduced density $\xi=\rho 2^{D} v_{0} / D\left(1-\chi^{2}\right)^{1 / 2}$, with $\chi=\left(k^{2}-1\right) /\left(k^{2}+1\right)$, is shown on a log-log plot for different values of the aspect ratio $k$ (equal to the ratio of the major to the minor axes of the ellipsoids). The isotropic branch is represented by a straight line which is independent of $k$. The nematic branch is shown for $k=2,3,4,5$, 6 , and $\infty$ (from top to bottom). The tie-line (also shown) indicates clearly how the isotropic-nematic transition is pushed to higher densities and also becomes narrower when $k$ decreases from the original Onsager limit $(k=\infty)$ to the spherical limit $(k=1)$ where the transition disappears.

TABLE II. The packing fraction $\eta=\rho v_{0}$ at the continuous isotropic-nematic transition of hard ellipses $(D=2)$ of aspect ratio $k$ as predicted by Eqs. (9) and (12) compared to the location of the transition (Kosterlitz-Thouless for $k=6$ and weakly first order for $k=4$ ) seen in the simulations of Cuesta and Frenkel [4]. The approximations are the same as in Table I: a one order-parameter approximation [11] for $h(\mathbf{u})$, the reduced Berne-Pechukas contact distance [4], and the hard-disk equation of state used in Ref. [11]. The superiority of Eq. (12) is seen from the fact that for $k=2$ the $I-N$ transition is again predicted to occur well above the isotropic-solid transition of the simulations (with the latter preempting the former). This time, however, the numerical agreement is rather poor pointing to a more complex relation between the radial and angular variables for $D=2$ than the simple factorization assumed here.

\begin{tabular}{lccc}
\hline \hline \multicolumn{1}{c}{$k$} & 6 & 4 & 2 \\
\hline MC & $\sim 0.59$ & $\sim 0.74$ & $(\sim 0.78)$ \\
Eq. (12) & 0.39 & 0.54 & 0.85 \\
Eq. (9) & 0.32 & 0.42 & 0.66 \\
\hline \hline
\end{tabular}


here, and elsewhere [11], from the close analogy between these generalized Onsager theories and the original Onsager [13] theory which also predicts [18] a continuous transition in $D=2$. Finally, the quantitative results obtained from (9) and (12) in $D=2$ are barely acceptable as illustrated in Table II. It could be argued [18] that for $D=2$ the convergence of the virial expansion is slower than for $D=3$ but this is an argument against the use of the original (low-density) Onsager theory in $D=2$ and not against the present finite-density generalizations of it which contain virial coefficients of all orders (although approximated here in terms of the HS virial coefficients). In conclusion, it is our impression that (12), when used in conjunction with a reasonable $\mathrm{HS}$ equation of state and a one order-parameter approximation for the angular distribution, will yield a good description of the $I-N$ transi- tion for $D \geq 3$ but that in order to remedy the qualitative and quantitative defects of (12) for $D=2$ the trivial factorization of the radial and angular variables as embodied in (7) will have to be abandoned.

This work has been partially supported by a grant from the Dirección General de Investigación Científica y Técnica (Spain) under Grant No. PB88-0140. One of us (M.B.) acknowledges the financial support of the Fonds National de la Recherche Scientifique and also from the Association Euratom-Etat Belge. Hong-Xu acknowledges the financial support of the Stichting voor Fundamenteel Onderzoek der Materie (FOM) of the Netherlands.
[1] See J. F. Lutsko and M. Baus, Phys. Rev. A 41, 6647 (1990), and references therein.

[2] See, e.g., B. B. Laird and D. M. Kroll, Phys. Rev. A 42, 4810 (1990); A. de Kuyper, W. L. Vos, J. L. Barrat, J. P. Hansen, and J. A. Schouten, J. Chem. Phys. 93, 5187 (1990); X. G. Wu and M. Baus, Mol. Phys. 62, 375 (1987); J. L. Barrat, J. P. Hansen, G. Pastore, and E. M. Waisman, J. Chem. Phys. 86, 6360 (1987).

[3] J. A. C. Veerman and D. Frenkel, Phys. Rev. A 41, 3237 (1990); A. Stroobants, H. N. W. Lekkerkerker, and D. Frenkel, ibid. 36, 2929 (1987); D. Frenkel and B. M. Mulder, Mol. Phys. 55, 1171 (1985); D. Frenkel, J. Phys. Chem. 92, 3280 (1988).

[4] J. A. Cuesta and D. Frenkel, Phys. Rev. A 42, 2126 (1990).

[5] U. P. Singh and Y. Singh, Phys. Rev. A 33, 2725 (1986).

[6] M. Baus, J. L. Colot, X. G. Wu, and H. Xu, Phys. Rev. Lett. 59, 2184 (1987); J. L. Colot, X. G. Wu, H. Xu, and M. Baus, Phys. Rev. A 38, 2022 (1988).

[7] J. F. Marko, Phys. Rev. Lett. 60, 325 (1988).
[8] S. D. Lee, J. Chem. Phys. 87, 4932 (1987); 89, 7036 (1988).

[9] A. Perera, G. N. Patey, and J. J. Weis, J. Chem. Phys. 89, 6941 (1988).

[10] R. Holyst and A. Poniewierski, Mol. Phys. 68, 381 (1989); Phys. Rev. A 39, 2742 (1989); A. Poniewierski and R. Holyst, Phys. Rev. Lett. 61, 2461 (1988).

[11] J. A. Cuesta, C. F. Tejero, and M. Baus, Phys. Rev. A 39, 6498 (1989).

[12] B. Tjipto-Margo and G. T. Evans, J. Chem. Phys. 93, 4254 (1990).

[13] L. Onsager, Ann. N.Y. Acad. Sci. 51, 627 (1949).

[14] R. Pynn, J. Chem. Phys. 60, 4579 (1974).

[15] P. Tarazona, Phys. Rev. A 31, 2672 (1985).

[16] J. A. Cuesta, C. F. Tejero, and M. Baus (unpublished).

[17] H. O. Carmesin, H. L. Frisch, and J. K. Percus, Phys. Rev. B 40, 9416 (1989).

[18] R. F. Kayser, Jr. and H. J. Raveché, Phys. Rev. A 17, 2067 (1978)

[19] J. A. Cuesta and C. F. Tejero, Phys. Lett. A 152, 15 (1991). 